Mortality risk in preterm and small-for-gestational-age infants in low-income and middle-income countries: a pooled country analysis. Lancet 2013; 382:417-25.

Correspondence: A. L. Slogrove, 1 Durban Street, Worcester, 6850, Western Cape, South Africa (amy@sun.ac.za).

Clinical Infectious Diseases ${ }^{\circledR} \quad$ 2019;68(12):2157-8

(C) The Author(s) 2018. Published by Oxford University Press for the Infectious Diseases Society of America. All rights reserved. For permissions, e-mail: journals.permissions@oup.com. DOI: $10.1093 /$ cid/ciy1056

\section{Reply to Slogrove et al}

We would like to thank Slogrove and colleagues for the positive comments on our manuscript and for emphasizing the need to provide definitive evidence of the benefit of controlling maternal human immunodeficiency virus (HIV) infections for the health of infants born in low- and middle-income countries (LMIC), where the burden of HIV infection is highest [1]. We agree that the pathways leading to the vulnerability of HIV-exposed, uninfected (HEU) infants may not be identical in LMIC and in high-income countries (HIC), and that the potential role of specific factors has to be determined. As proposed by Slogrove and colleagues, the decreased risk of hospitalization observed in our study for those infections associated with the initiation of antiretroviral therapy (ART) before pregnancy may be offset by an increased risk of premature delivery in women living in LMIC [2]. On the other hand, contrary to HIC, women living with HIV in LMIC are encouraged to breastfeed. Although the evidence from HIC is less consistent [3], there is strong supportive evidence for a protective effect of breastfeeding on infectious morbidity in LMIC [4]. Through a diversity of immunological components, breastfeeding could reduce the immunological risk of severe infections after birth and thereby mitigate the impact of immune alterations induced by in utero exposure to maternal HIV infection. In our study, maternal and newborn immune activation predicted the risk of hospitalization due to infection in infants born to mothers who initiated ART during pregnancy [5]. Immune activation is commonly observed in adults living in LMIC, independently of HIV infection [6]. Therefore, the potential for ART to correct immune activation in women living with HIV may be lower in LMIC as compared to HIC, and this could mitigate the impact of ART initiation before pregnancy on infants' susceptibility to infectious diseases.

Although the vulnerability of HEU infants living in different settings could involve different factors, it is essential to recognize that this vulnerability is a global public health issue. An increased susceptibility of HEU infants to severe infections is observed in both LMIC and HIC, suggesting that common determinants are playing a critical role $[7,8]$. Identifying these determinants has the potential to positively impact the health of HEU infants worldwide. To meet this challenge, researchers in HIC and LMIC should join efforts and integrate both intensive studies on relatively small study populations and larger studies that are powered to determine the impact of key environmental factors on clinical outcomes. Control of maternal HIV infection before pregnancy and progress in our understanding of the immunobiology of infant exposure to maternal HIV infection provide unprecedented opportunities to further improve the health of children born to HIV-infected mothers.

\section{Note}

Potential conflicts of interest. A. M.s institution has received fees from GlaxoSmithKline Vaccines, outside the submitted work. G. A.'s institution has received grants from Gilead Sciences and the Bill and Melinda Gates Foundation (grant numbers OPP1032817, OPP1097381, and OPP1114729). T. R. K.'s institution has received grants from the National Institute for Allergy and Infectious Diseases and the Canada Institutes for Health Research. All other authors report no potential conflicts. All authors have submitted the ICMJE Form for Disclosure of Potential Conflicts of Interest. Conflicts that the editors consider relevant to the content of the manuscript have been disclosed.

Tessa Goetghebuer, ${ }^{1}$ Kinga K. Smolen, Catherine Adler, ${ }^{1}$ Jishnu Das, ${ }^{3,4}$ Trevor McBride, Gaby Smits, ${ }^{6}$ Sandra Lecomte, ${ }^{2}$ Edwige Haelterman, ${ }^{1}$ Patricia Barlow, ${ }^{7}$ Pedro A. Piedra, ${ }^{5,6}$ Fiona van der Klis, ${ }^{8}$ Tobias R. Kollmann, ${ }^{9}$ Douglas A. Lauffenburger, ${ }^{4}$ Galit Alter, ${ }^{3}$ Jack Levy, ${ }^{1}$ and Arnaud Marchant ${ }^{2}$

'Department of Pediatrics, Hôpital Saint-Pierre, Brussels, and ${ }^{2}$ Institute for Medical Immunology, Université libre de Bruxelles, Charleroi, Belgium; ${ }^{3}$ Ragon Institute of Massachusetts General Hospital, Massachusetts Institute of Technology and Harvard University, and ${ }^{4}$ Department of Biological Engineering. Massachusetts Institute of

Technology, Cambridge, Massachusetts; ${ }^{5}$ Department of Molecular Virology and Microbiology, and ${ }^{6}$ Department of

Pediatrics, Baylor College of Medicine, Houston, Texas; ${ }^{7}$ Department of Obstetrics and Gynecology, Hôpital Saint-

Pierre, Brussels, Belgium; ${ }^{8}$ National Institute of Health and the Environment, Bilthoven, The Netherlands; and ${ }^{9}$ Division of Infectious Diseases, Department of Pediatrics, University of British Columbia, Vancouver, Canada

\section{References}

1. Slogrove AL, Powis KM, Cotton MF. HIV exposed uninfected infants: surviving and thriving or overlooked by success? Clin Infect Dis. 2018 Dec 11 [Epub ahead of print]. doi:10.1093/cid/ciy1056

2. Uthman OA, Nachega JB, Anderson J, et al. Timing of initiation of antiretroviral therapy and adverse pregnancy outcomes: a systematic review and meta-analysis. Lancet HIV 2017; 4:e21-30.

3. Størdal K, Lundeby KM, Brantsæter AL, et al. Breast-feeding and infant hospitalization for infections: large cohort and sibling analysis. J Pediatr Gastroenterol Nutr 2017; 65:225-31.

4. Victora CG, Bahl R, Barros AJ, et al; Lance Breastfeeding Series Group. Breastfeeding in the 21st century: epidemiology, mechanisms, and lifelong effect. Lancet 2016; 387:475-90.

5. Goetghebuer T, Smolen KK, Adler C, et al. Initiation of anti-retroviral therapy before pregnancy reduces the risk of infection-related hospitalization in HIV-exposed uninfected infants born in a high-income country. Clin Infect Dis 2018.

6. Marchant A, Sadarangani M, Garand M, et al. Maternal immunisation: collaborating with mother nature. Lancet Infect Dis 2017; 17:e197-208

7. Slogrove AL, Goetghebuer T, Cotton MF, Singer J, Bettinger JA. Pattern of infectious morbidity in HIV-exposed uninfected infants and children. Front Immunol 2016; 7:1-8.

8. Ruck C, Reikie BA, Marchant A, Kollmann TR, Kakkar F. Linking susceptibility to infectious diseases to immune system abnormalities among HIV-exposed uninfected infants. Front Immunol 2016; 7:1-12. 
Correspondence: A Marchant, Institute for Medical Immunology, Université Libre de Bruxelles Gosselies, Rue Adrienne Bolland 8, B-6041 Gosselies, Belgium (Arnaud. Marchant@ulb.ac.be).

Clinical Infectious Diseases ${ }^{\circledR} \quad$ 2019;68(12):2158-9 (C) The Author(s) 2018. Published by Oxford University Press for the Infectious Diseases Society of America. All rights reserved. For permissions, e-mail: journals.permissions@oup.com. DOI: $10.1093 /$ cid/ciy1058

\section{Does Tenofovir-containing First-line Antiretroviral Therapy Mitigate the Impact of Pretreatment Non-nucleoside Reverse Transcriptase Inhibitor Drug Resistance?}

To THE EdiTOR-In a human immunodeficiency virus treatment-as-prevention trial in South Africa, Derache et al [1] report the remarkable finding that the presence of non-nucleoside reverse transcriptase inhibitor (NNRTI)-associated pre-treatment drug resistance (PDR) did not impair virological responses to fixeddose tenofovir/emtricitabine/efavirenz (Atripla). This is an important contrast with most past studies, which found that NNRTI-associated PDR was associated with a 2- to 3-fold increased risk of virological failure (VF) [2-9], although most patients in those studies received a thymidine analogue backbone (zidovudine/ stavudine) with efavirenz or nevirapine $[2,3]$. The authors attributed their finding to the better potency of efavirenz compared to nevirapine, and an Editorial Comment added the advantage of the similar half-lives of the Atripla components, making it less likely for resistance to emerge during missed doses [10].

In our previous analysis in the PanAfrican Studies to Evaluate Resistance (PASER-M) cohort, we reported that patients with NNRTI-PDR had a $>2$-fold increased risk of VF, compared to patients with a susceptible virus $[4,7]$. Based on the hypothesis by Derache et al [1], we extended this to a stratified analysis by type of first-line regimen. We defined PDR as (1) an NNRTI, nucleoside reverse transcriptase inhibitor (NRTI), or dual-class NNRTI+NRTI resistance, based on the 2017 International Antiviral Society (IAS)USA mutation list; or (2) a Stanford genotype susceptibility score (GSS; v8.7) $<3$ of the prescribed first-line regimen. We defined VF as a single viral load (VL) $\geq 50$, 400 , or $1000 \mathrm{cps} / \mathrm{ml}$, measured at month 12 . We assessed the association between PDR and VF using logistic regression, while adjusting for key confounders.

Of 2737 participants initiating first-line antiretroviral therapy (ART), 1941 had data on PDR and the 12 month VL. The median age was 37.0 years (interquartile range $31.7-43.1$ ), 59.8\% were women, and 56.4\% had an overall mean adherence of $\geq 95 \%$ (as measured by visual analogue scale). Initial regimens contained either tenofovir and lamivudine/emtricitabine (xtc; 33\%), with efavirenz $(27.3 \%)$ or nevirapine $(5.7 \%)$, or a non-tenofovir, thymidine analogue backbone and xtc (67\%), with efavirenz $(29.8 \%)$ or nevirapine (37.1\%). In all, 1838 (94.7\%) patients had no PDR, 79 (4.1\%) had NNRTIPDR only, 44 (2.3\%) had NRTI-PDR, and 24 (1.2\%) had dual-class NNRTI+NRTI-PDR. Only 84 (4.4\%) patients initiated a first-line regimen with GSS <3. VF was present in 335 (17.3\%), 199 (10.3\%), and 172 (8.9\%) participants at VL thresholds $\geq 50,400$, and $1000 \mathrm{cps} / \mathrm{ml}$, respectively.

Participants who had PDR defined as GSS < 3, NNRTI only, or dual-class NNRTI+NRTI who received non-tenofovir/xtc with efavirenz or nevirapine had an increased risk of VF, compared to those without PDR. However, this risk was not increased for participants who received tenofovir/xtc/efavirenz, whereas there was a borderline association for participants who received tenofovir/xtc/nevirapine (Table 1). Participants with NNRTI-PDR only who received a tenofovir-containing regimen had an increased risk of $\mathrm{VF}$ at the VL threshold of $\geq 1000 \mathrm{cps} / \mathrm{ml}$ (with borderline statistical significance of $P=.073$ ), and the risk was not increased at the $\geq 50$ and $\geq 400 \mathrm{cps} / \mathrm{ml}$ thresholds.

In conclusion, our analysis corroborates the finding that NNRTI-PDR may impact less on tenofovir/xtc/efavirenz than on thymidine analogue-based regimens, especially with nevirapine. Nonetheless, it remains difficult to disentangle the possible beneficial effects of tenofovir, efavirenz, and fixed-dose combinations with similar drug half-lives. Given that 2 other studies have produced conflicting data $[2,3]$, it is premature to argue that Atripla is equally efficacious for patients with or without NNRTI-PDR.

\section{Notes}

Author contributions. T. F. R. dW. is the PanAfrican Studies to Evaluate Resistance principal investigator. R. L. H. and T. F. R. dW. conceived the study. S. C. I. performed the statistical analysis. S. C. I. and R. L. H. drafted the manuscript. All authors provided valuable input to the interpretation of the data and critically reviewed the paper for important intellectual content. All authors reviewed and approved the final version of the manuscript.

Acknowledgments. The authors thank the study participants, the staff at the collaborating clinical sites, and the reference laboratories.

Disclaimer. Pan-African Studies to Evaluate Resistance is part of the Linking African and Asian Societies for an Enhanced Response to Human Immunodeficiency Virus/Acquired Immunodeficiency Syndrome (LAASER) program, a partnership of Stichting Aids Fonds, the Foundation for Acquired Immunodeficiency Syndrome Research (amfAR)-Therapeutics Research, Education, and AIDS Training in Asia, PharmAccess Foundation, and International Civil Society Support.

Financial support. This work was supported by the Pan-African Studies to Evaluate Resistance, an initiative of the Amsterdam Institute for Global Health and Development, with major support provided by the Ministry of Foreign Affairs of the Netherlands through a partnership with Stichting Aids Fonds (grant number 12454) and The Netherlands Organization for Scientific Research (NWO-WOTRO grant numbers W07.10.101 and W07.10.106), and additional support provided by De Grote Onderneming, The Embassy of the Kingdom of the Netherlands, Heineken Africa Foundation, and Jura Foundation.

Potential conflicts of interest. All authors: No reported conflicts of interest. All authors have submitted the ICMJE Form for Disclosure of Potential Conflicts of Interest. Conflicts that the editors consider relevant to the content of the manuscript have been disclosed.

Seth C. Inzaule, ${ }^{1,2, \odot}$ Tobias F. Rinke de Wit, ${ }^{1,2}$ and Raph L. Hamers ${ }^{1,3,4,5}$; on behalf of Pan-African Studies to Evaluate Resistance

${ }^{1}$ Amsterdam Institute for Global Health and Development, Department of Global Health, Amsterdam University Medica Center, University of Amsterdam, and ${ }^{2}$ Joep Lange Institute, Amsterdam, The Netherlands; ${ }^{3}$ Eijkman-Oxford Clinical

Research Unit, Eijkman Institute for Molecular Biology, and ${ }^{4}$ Faculty of Medicine, Universitas Indonesia, Jakarta; and

${ }^{5}$ Center for Tropical Medicine and Global Health, Nuffield Department of Medicine, University of Oxford, United Kingdom

\section{References}

1. Derache A, Iwuji CC, Baisley K, et al. Impact of next generation sequencing defined HIV pre-treatmen 